\title{
Many-Body Quantum Spin Dynamics with Monte Carlo Trajectories on a Discrete Phase Space
}

\begin{abstract}
J. Schachenmayer, A. Pikovski, and A. M. Rey
JILA, NIST and Department of Physics, University of Colorado, 440 UCB, Boulder, Colorado 80309, USA (Received 14 August 2014; revised manuscript received 8 October 2014; published 25 February 2015)

Interacting spin systems are of fundamental relevance in different areas of physics, as well as in quantum information science and biology. These spin models represent the simplest, yet not fully understood, manifestation of quantum many-body systems. An important outstanding problem is the efficient numerical computation of dynamics in large spin systems. Here, we propose a new semiclassical method to study many-body spin dynamics in generic spin lattice models. The method is based on a discrete Monte Carlo sampling in phase space in the framework of the so-called truncated Wigner approximation. Comparisons with analytical and numerically exact calculations demonstrate the power of the technique. They show that it correctly reproduces the dynamics of one- and two-point correlations and spin squeezing at short times, thus capturing entanglement. Our results open the possibility to study the quantum dynamics accessible to recent experiments in regimes where other numerical methods are inapplicable.
\end{abstract}

DOI: 10.1103/PhysRevX.5.011022

\section{INTRODUCTION}

Controlled experimental observation of nonequilibrium spin dynamics has recently become possible [1-3]. Large spin systems with long-range interactions have been realized, e.g., with polar molecules [4,5], Rydberg atoms [6-8], and trapped ions [9-12]. Key aspects of quantum dynamics, such as the buildup of long-range correlations, entanglement, and the propagation of information, are insufficiently understood, partly due to the absence of appropriate tools to calculate the time evolution in complex quantum systems.

Current techniques are not suitable for the investigation of quantum dynamics in generic large spin systems. For example, numerical time-dependent density matrix renormalization group (tDMRG) methods [13-15] become inefficient in higher dimensional systems; perturbative and Keldysh techniques [16], as well as kinetic theories and phase space methods [17-19], are limited to weakly interacting, close-to-equilibrium or short-time situations; cluster expansions [5] work only for dilute samples with moderately short-ranged interactions. The development of new numerical techniques is, therefore, of immediate relevance. In this work, we advance in this direction by introducing a semiclassical phase-space method that we refer to as the discrete truncated Wigner approximation (DTWA). With this relatively easily programable method, we can calculate nonequilibrium dynamics in systems of thousands of spins and in arbitrary dimensions.

Published by the American Physical Society under the terms of the Creative Commons Attribution 3.0 License. Further distribution of this work must maintain attribution to the author(s) and the published article's title, journal citation, and DOI.
Subject Areas: Quantum Physics, Statistical Physics

The DTWA is a semiclassical method which is based, in contrast to existing techniques, on the sampling of a discrete Wigner function. Standard phase-space methods, such as the truncated Wigner approximation (TWA), replace the quantum-mechanical time evolution by a semiclassical evolution via classical trajectories. The quantum uncertainty in the initial state is accounted for by an average over different initial conditions $[17,18]$ determined by a continuous Wigner function. In contrast, the use of discrete Wigner functions enables us to quantitatively access dynamics in generic spin lattice models, including oscillations and revivals of single particle observables and correlation functions that are not captured by the TWA.

This paper is organized as follows: In Sec. II A, the traditional TWA is reviewed, and in Sec. II B, our DTWA technique is introduced. Section III contains a benchmark of the improvement provided by the DTWA via comparisons of dynamics of single-spin observables, correlation functions, and spin squeezing for a model with Ising and $X Y$ interactions. Section IV provides a conclusion and an outlook.

\section{METHOD}

\section{A. Semiclassical phase-space sampling}

The mapping between the Hilbert space of a quantum system and its corresponding phase space (known as the Wigner-Weyl transform) can be accomplished through the so-called phase-point operators $\hat{A}$. In terms of classical phase-space variables $\mathbf{p}$ and $\mathbf{q}$ (we set $\hbar=1$ in this paper), the phase-point operators can be written as $[20,21]$ $\left\langle\mathbf{q}^{\prime}|\hat{A}(\mathbf{p}, \mathbf{q})| \mathbf{q}^{\prime \prime}\right\rangle=\left[1 /(2 \pi)^{D}\right] \delta\left\{\mathbf{q}-\left[\left(\mathbf{q}^{\prime}+\mathbf{q}^{\prime \prime}\right) / 2\right]\right\} e^{i \mathbf{p} \cdot\left(\mathbf{q}^{\prime}-\mathbf{q}^{\prime \prime}\right)}$ with $D$ the phase-space dimension. They relate the density 
matrix of the quantum system $\hat{\rho}$ to a quasiprobability distribution (generally nonpositive) known as the Wigner function $W$ [21], which is given by $W(\mathbf{p}, \mathbf{q})=$ $\operatorname{Tr}[\hat{\rho} \hat{A}(\mathbf{p}, \mathbf{q})]$. Any operator $\hat{O}(\mathbf{p}, \mathbf{q})$ can be mapped to a function over the classical phase space, the so-called Weyl symbol $\mathcal{O}_{W}(\mathbf{p}, \mathbf{q})=\operatorname{Tr}[\hat{O} \hat{A}(\mathbf{p}, \mathbf{q})]$. To compute the time evolution of the expectation value of an operator, its timeevolved Weyl symbol has to be averaged over the phase space with the corresponding Wigner function: $\langle\hat{O}\rangle(t)=$ $\iint d \mathbf{p} d \mathbf{q} \mathcal{O}_{W}(\mathbf{p}, \mathbf{q}) W(\mathbf{p}, \mathbf{q} ; t)$. In general, however, it is not possible to compute the time evolution exactly. The TWA $[17,18]$ approximates the dynamics by taking only firstorder quantum fluctuations into account. In the Heisenberg picture, the Wigner function is fixed to its initial state $W(\mathbf{p}, \mathbf{q}) \rightarrow W\left(\mathbf{p}_{0}, \mathbf{q}_{0}\right)$ and the Weyl symbol evolves in time. The TWA makes the approximation that the Weyl symbols follow a classical evolution. They are obtained by solving Hamilton's equations of motion for $\mathbf{p}_{c l}(t), \mathbf{q}_{c l}(t)$ with the initial conditions $\left(\mathbf{p}_{0}, \mathbf{q}_{0}\right)$, and one puts $\mathcal{O}_{W}(\mathbf{p}, \mathbf{q})(t) \rightarrow \mathcal{O}_{W}\left(\mathbf{p}_{c l}(t), \mathbf{q}_{c l}(t)\right) ;$ thus,

$$
\langle\hat{O}\rangle(t) \approx \iint d \mathbf{p}_{0} d \mathbf{q}_{0} \mathcal{O}_{W}\left(\mathbf{p}_{c l}(t), \mathbf{q}_{c l}(t)\right) W\left(\mathbf{p}_{0}, \mathbf{q}_{0}\right) .
$$

Generalizations of this continuous formulation to $N$ spin$1 / 2$ particles have been developed, e.g., by means of a spincoherent state representation, valid up to $1 / N$ corrections. Generically, the Wigner function is approximated by a smooth positive Gaussian-like distribution in the collective spin variables. For example, if all spins are pointing along the $z$ axis, the Wigner function can be approximately written as [19] $W\left(S_{\perp}, S_{z}\right) \approx 1 /(\pi S) e^{-S_{\perp}^{2} / S} \delta\left(S_{z}-S\right)$, with $S=N / 2$, $S_{\perp}=\left(S_{x}^{2}+S_{y}^{2}\right)^{1 / 2}$, and $S_{z}$ the transverse and longitudinal spin components of the collective classical spin, respectively. This Wigner function has a clear interpretation: If each spin initially points along the $z$ direction, the transverse spin components must fluctuate as $\left\langle S_{\perp}^{2}\right\rangle \sim S$ due to the Heisenberg uncertainty principle.

This Gaussian TWA generally captures aspects of the quantum spin dynamics at short times but lacks important ones, such as the revivals [see, e.g., Fig. 2(a)], ubiquitous in discrete quantum systems, and it is mainly limited to dealing with the collective dynamics [18]. For interactions with spatial structure, the dynamics quickly takes the system out of the collective-spin Hilbert space and the TWA breaks down. In the continuous phase-space picture, ways to overcome these shortcomings have been proposed using hidden variables [22] or more complex representations of the Wigner function [18,23,24]. Here, we propose and test a different approach, which uses discrete phase spaces for each individual spin in conjunction with a Monte Carlo sampling.

\section{B. Discrete phase-space sampling}

For systems with discrete degrees of freedom, it is possible to define a "discrete phase space" in many ways (see Ref. [20] and references therein). We use four phase points to describe a single spin- $1 / 2$, which we denote as $\alpha \equiv(q, p) \in\{(0,0),(0,1),(1,0),(1,1)\}$, as introduced by Wootters $[20,25]$. For each phase-space point, one can define a phase-point operator $\hat{A}_{\alpha}$, the Weyl symbols $\mathcal{O}_{\alpha}^{W}=\operatorname{tr}\left(\hat{O} \hat{A}_{\alpha}\right) / 2$ (or inversely $\left.\hat{O}=\sum_{\alpha} \hat{A}_{\alpha} \mathcal{O}_{\alpha}^{W}\right)$, and the Wigner function $w_{\alpha}$, which is the Weyl symbol for the density matrix. A set of phase-point operators is given by [20]

$$
\hat{A}_{\alpha}=\hat{\wp}\left(\mathbf{r}_{\alpha}\right), \quad \hat{\wp}(\mathbf{r}) \equiv(1+\mathbf{r} \cdot \hat{\boldsymbol{\sigma}}) / 2,
$$

with $\mathbf{r}_{(0,0)}=(1,1,1), \mathbf{r}_{(0,1)}=(-1,-1,1), \mathbf{r}_{(1,0)}=(1,-1,-1)$, and $\mathbf{r}_{(1,1)}=(-1,1,-1)$. Here, $\hat{\boldsymbol{\sigma}}=\left(\hat{\sigma}^{x}, \hat{\sigma}^{y}, \hat{\sigma}^{z}\right)$ are the Pauli matrices. In a many-body system with $N$ spin- $1 / 2$ particles, the discrete phase space has $4^{N}$ points we denote as $\alpha=\left\{\alpha_{1}, \alpha_{2}, \ldots, \alpha_{N}\right\}$. Analogously to Eq. (1), we can now formulate the DTWA on this discrete phase space as

$$
\langle\hat{O}\rangle(t)=\sum_{\boldsymbol{\alpha}} w_{\alpha}(0) \mathcal{O}_{\boldsymbol{\alpha}}^{W}(t) \approx \sum_{\boldsymbol{\alpha}} w_{\alpha}(0) \mathcal{O}_{\boldsymbol{\alpha}}^{W, \mathrm{cl}}(t),
$$

where $w_{\alpha}(0)$ is the initial Wigner function on the discrete many-body phase space and $\mathcal{O}_{\alpha}^{W, c l}(t)$ is the classically evolved Weyl symbol (see Appendix A for more on the classical equations of motion). Numerically, we can solve Eq. (3) by statistically choosing [according to $w_{\alpha}(0)$ ] a large number $n_{t}$ of random initial spin configurations. Each "trajectory" is evolved in time according to the classical equations of motion, and the expectation value in Eq. (3) is estimated via statistical averaging (error $\sim 1 / \sqrt{n_{t}}$ ). We find that the required $n_{t}$ does not depend on the system size, but rather on the observable under consideration (see also Appendix C).

As an example of how to construct the initial Wigner function, we again consider an initial state with all spins pointing along the $z$ direction. The initial density matrix factorizes $\hat{\rho}(0)=\prod_{i=1}^{N} \hat{\wp}^{[i]}(\hat{z})$ (the superscript $[i]$ denotes the Hilbert or phase space for spin $i$ ), and thus, $w_{\alpha}(0)=\Pi_{i=1}^{N} w_{\alpha_{i}}^{[i]}$. Here, $w_{\alpha_{i}}^{[i]}=\operatorname{Tr}\left[\hat{\wp}^{[i]}(\hat{z}) \hat{A}_{\alpha_{i}}\right] / 2$ is given by $w_{(0,0)}^{[i]}=w_{(0,1)}^{[i]}=1 / 2$, and $w_{(1,0)}^{[i]}=w_{(1,1)}^{[i]}=0$ for every spin $i$ (cf. Fig. 1 for an illustration). Note that for this initial state, all quasiprobabilities are positive, which is important for the numerical sampling. The sum along each "phasespace line" of the discrete Wigner function can be associated with the probability of a measurement outcome [20], similarly to the continuous variable case. As shown in Fig. 1, here $w_{(0,0)}^{[i]}=w_{(0,1)}^{[i]}=1 / 2$ means that the probability to find an individual spin pointing along the $+z$ direction is 1 (sum over the first row), while the probabilities to find it along $+x$ or $-x$ (sum over each column) are $50 \%$ each. Equally, the probabilities to find it along $+y$ or $-y$ are $50 \%$ each (sum over each of the two diagonals). 

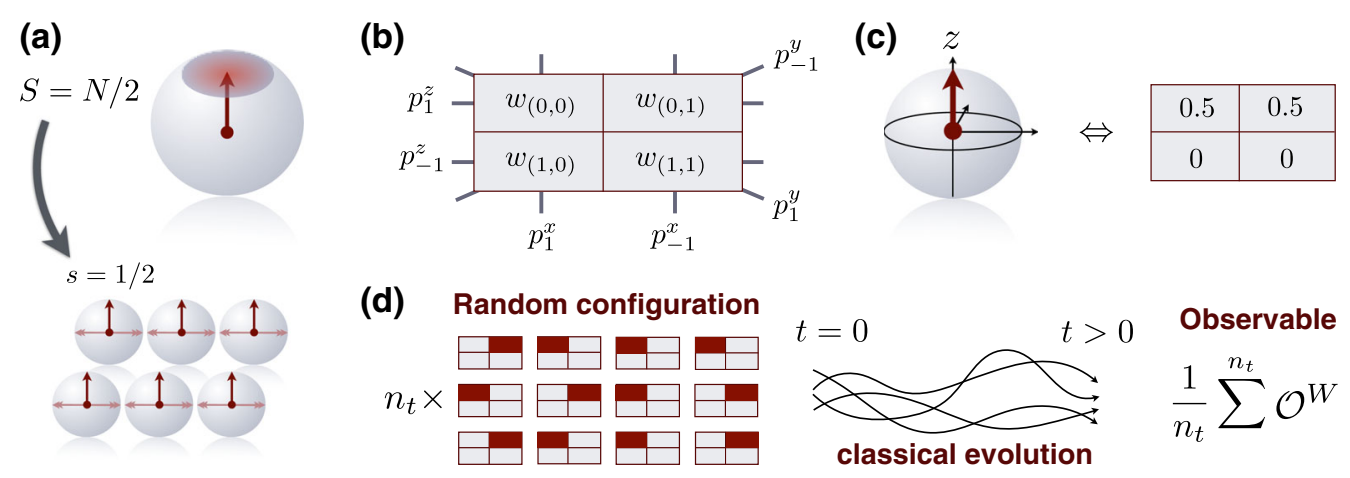

FIG. 1. Discrete phase space and the DTWA. (a) Our method considers the quantum uncertainties of $N$ spin- $1 / 2$ particles individually, rather than the noise of the collective spin $S=N / 2$. (b) The quantum physics of a spin-1/2 particle can be fully described by a discrete four-point Wigner quasiprobability distribution, $w_{(p, q)}$. The probability for a spin to point along the $\pm x, \pm y$, and $\pm z$ directions $\left(p_{ \pm 1}^{x, y, z}\right)$ is given by the sum over the vertical, diagonal, and horizontal lines in phase space, respectively [20]. (c) Discrete Wigner function of a spin pointing along $z$. (d) The idea behind the DTWA is to (i) randomly sample the phase points for each spin according to $w_{(p, q)}$, (ii) calculate the time evolution according to classical equations, and (iii) evaluate observables in phase space from the statistical mixture of $n_{t}$ trajectories.

Note that this discrete sampling properly accounts for the quantum-mechanical probability distribution of the $x, y, z$ spin components of a qubit in the sense that all moments are reproduced correctly: $\left\langle\left(\hat{\sigma}^{x, y}\right)^{k}\right\rangle=\left[1+(-1)^{k}\right] / 2,\left\langle\left(\hat{\sigma}^{z}\right)^{k}\right\rangle=1$, with $k$ a positive integer.

\section{DYNAMICS USING THE DTWA}

To demonstrate the accuracy of the DTWA, we consider a system of $N$ two-level systems arranged on a lattice with $M$ sites with dynamics governed by the Hamiltonian

$\hat{H}=\frac{1}{2} \sum_{i \neq j}\left[\frac{J_{i j}^{\perp}}{2}\left(\hat{\sigma}_{i}^{x} \hat{\sigma}_{j}^{x}+\hat{\sigma}_{i}^{y} \hat{\sigma}_{j}^{y}\right)+J_{i j}^{z} \hat{\sigma}_{i}^{z} \hat{\sigma}_{j}^{z}\right]+\Omega \sum_{i} \hat{\sigma}_{j}^{x}$.

We consider an initial product state in which all spins are aligned along the $x$ axis. The interactions are assumed to decay as a function of the distance with a decay exponent $\alpha$ and are allowed to be spatially inhomogeneous: e.g., $J_{i j}^{\perp / z} \equiv J\left[1-3 \cos (\theta)^{2}\right] /\left|\mathbf{r}_{i j}\right|^{\alpha}$. Here, $\mathbf{r}_{i j}$ is the vector connecting spins on sites $i$ and $j$, and $\theta$ is the angle it makes with the quantization axis (chosen along $z$ ). We discuss two specific cases: Ising $\left(J_{i j}^{\perp}=0\right)$ and $X Y \quad\left(J_{i j}^{z}=0\right)$ interactions. In addition to the interactions, we allow for a transverse field with strength $\Omega$. The Ising limit is naturally realized in experiments with ion traps (both in 1D [10,11] or 2D [12] geometries) and Rydberg atoms in 2D [8]; the $X Y$ limit dynamics have been realized in polar molecules in optical lattices [4,5], magnetic atoms [26], Förster resonances in Rydberg atoms [27], and as an effective Hamiltonian in trapped ions with a superimposed large transverse field [11].

The classical equations of motion (for classical spin components $s_{i}^{x, y, x}$ ) corresponding to Hamiltonian (4) are given in Appendix A. The DTWA method simply consists of a numerical integration of the classical equations of motion for many different random initial conditions. While for each site $i$, the initial condition of the classical spin component along $x, s_{i}^{x}=1$ is fixed, the initial conditions for the spin components in the orthogonal directions are randomly chosen as $s_{i}^{y}, s_{i}^{z}= \pm 1$ (as shown in Sec. II B). The final expectation values of observables are calculated by averaging the results for the corresponding observable over all initial conditions, i.e., all trajectories.

\section{A. Ising interactions}

We now consider Ising interactions, $J_{i j}^{\perp}=0, \Omega=0$, in Eq. (4). In this limit, exact analytical expressions for the dynamics exist [28-30] and can be used to benchmark the DTWA. The dynamics of observables involving the collective spin $\boldsymbol{S}=\left(\left\langle S_{x}\right\rangle,\left\langle S_{y}\right\rangle,\left\langle S_{z}\right\rangle\right)^{T} \equiv \sum_{n}\left\langle\hat{\boldsymbol{\sigma}}_{n}\right\rangle$ for a system with $N=100$ spins in a one-dimensional chain with $M=100$ sites (oriented along $x$ ) are shown in Fig. 2. We calculate the time evolution of $S_{x}$ as well as the collective correlation functions $\Delta S_{x}=\left\langle S_{x}^{2}\right\rangle-\left\langle S_{x}\right\rangle^{2}$ and $\operatorname{Re}\left\langle S_{y} S_{z}\right\rangle$. We consider the case of all-to-all (decay exponent $\alpha=0$ ) and short-ranged dipolar interactions $(\alpha=3)$. In the all-toall case, $\left\langle S_{x}\right\rangle$ shows revivals at times that are multiples of $\pi / 2 J$. In contrast to the traditional Gaussian TWA, which captures only the initial decay of $\left\langle S_{x}\right\rangle$ and misses the revivals, the DTWA fully reproduces the exact dynamics (a similar effect has been seen in Ref. [31]). The case $\alpha=3$ exhibits an oscillatory dynamics, perfectly accounted for by the DTWA solution, but not captured by the traditional TWA. The DTWA calculations for $\operatorname{Re}\left\langle S_{y} S_{z}\right\rangle$ also show perfect agreement. Deviations are visible for the correlation $\Delta S_{x}$; however, the oscillatory dynamics is still better reproduced in the DTWA than in the traditional TWA.

We understand the agreement between the Ising solution and the DTWA analytically. For a particular spin $n$, the exact solution for the time evolution is $\left\langle\hat{\sigma}_{n}^{x}\right\rangle(t)=$ $\prod_{i \neq n}^{N} \cos \left(2 t J_{i n}^{z}\right)=\sum_{\mathbf{m}} \cos \left[2 t \sum_{a=1}^{N}\left(J_{n a}^{z} m_{a}\right)\right] / 2^{N}$, where $\mathbf{m}=\left\{m_{1}, m_{2}, \ldots, m_{N}\right\}$ and each of the $m_{a}$ takes the 

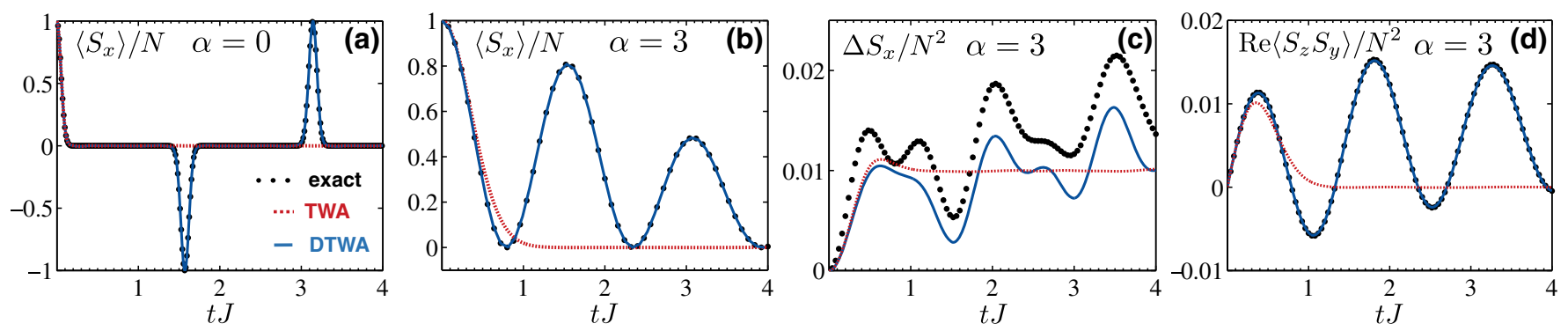

FIG. 2. Dynamics for Ising interactions. Circles denote the exact solution, dashed lines are traditional TWA results, solid lines denote DTWA results, for $1 \mathrm{D}, N=100$ spins. (a),(b) Evolution of $\left\langle S_{x}\right\rangle$, for all-to-all (decay exponent $\alpha=0$ ) and dipolar $(\alpha=3$ ) interactions, respectively. Traditional TWA captures only the initial decay and no oscillations or revivals. In contrast, DTWA becomes exact (on top of the black symbols). (c),(d) The evolution of the correlation functions $\Delta S_{x}=\left\langle S_{x}^{2}\right\rangle-\left\langle S_{x}\right\rangle^{2}$ and $\operatorname{Re}\left\langle S_{y} S_{z}\right\rangle$ for dipolar interactions. While $\operatorname{Re}\left\langle S_{y} S_{z}\right\rangle$ is exactly captured in DTWA, $\Delta S_{x}$ shows deviations. DTWA improves traditional TWA predictions in all panels.

values \pm 1 . The classical equation of motion for the $x$ component of spin is $s_{n}^{x}(t)=\cos \left(2 t \beta_{n}\right)$ (see Appendix A), where $\beta_{n}=\sum_{i \neq n}^{N} J_{i n} s_{i}^{z}(t=0)$. Since we initially sample the $z$ component of the spin to be randomly distributed as $s_{i}^{z}(t=0)= \pm 1$, we can identify $s_{i}^{z}(t=0)$ with the parameters $m_{i}$ in the exact solution and find that the DTWA becomes equivalent to the exact solution for large $n_{t}$ and arbitrary numbers of spins $N$. Note that the traditional TWA approach is valid only in the large- $N$ limit. The comparisons with the exact solution, extended to correlation functions, confirm the excellent agreement of $\operatorname{Re}\left\langle S_{y} S_{z}\right\rangle$ and show the origin of the discrepancies in other two-point correlations (see Appendix B and Fig. 2).

\section{B. $X Y$ model}

Next, we consider the dynamics for the $X Y$ model, $J_{i j}^{z}=0, \Omega=0$, in Eq. (4). Here, we study systems with varying filling fractions $\bar{n}=N / M$, a situation relevant for recent polar molecule experiments [4]. We focus our attention on the time evolution of spin squeezing, which is a signature of quantum correlations and entanglement
[32] and is a resource for enhanced sensitivity in quantum metrology [33]. The spin-squeezing parameter is $\xi \equiv \sqrt{N} \min _{\mathbf{n}_{\perp}}\left(\Delta S_{\perp}\right) /|\mathbf{S}|$, where $\mathbf{S}$ is the total collective spin, $S_{\perp}=\mathbf{S} \cdot \mathbf{n}_{\perp}$, and the minimum is taken over all unit vectors $\mathbf{n}_{\perp}$ (directions) perpendicular to the vector $\mathbf{S}$. On the Bloch sphere, a squeezed state with $\xi<1$ shows an elliptical profile of the spin noise distribution [34,35].

For the $X Y$ model, no exact solution exists for generic spin systems; however, in 1D we can use tDMRG to calculate the exact dynamics at short times. This technique works as long as the bipartite entanglement in the system, quantified by the half-chain von Neumann entropy $S_{\mathrm{vN}}\left(\rho_{L}\right)=-\operatorname{tr}\left(\rho_{L} \log _{2} \rho_{L}\right)$ (where $\rho_{L}$ is the reduced density matrix of half the spin chain), remains small [36-39]. Surprisingly, we find that for intermediate filling fractions, due to the inhomogeneity in $J_{i j}^{\perp}$, the entropy $S_{\mathrm{vN}}$ grows much more rapidly than for $\bar{n}=1$ [see inset in Fig. 3(b)]. Thus, with reasonable computational resources, exact results could be calculated for only $N \leq 32$ and $N=$ 100 spins in a system with $M=100$ sites.
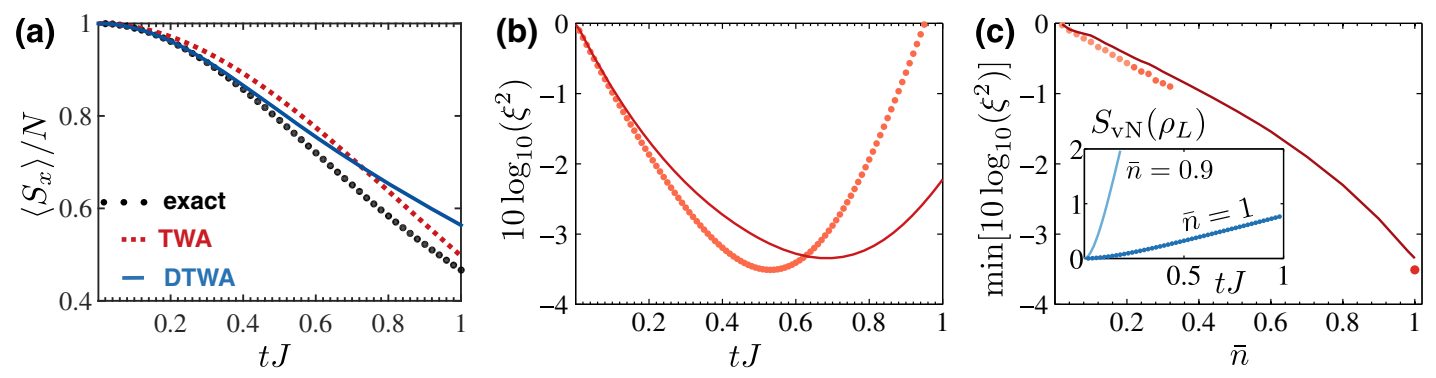

FIG. 3. Dynamics for $X Y$ interactions. Circles denote exact, solid lines DTWA, and dotted lines traditional TWA results. (a) Time evolution of $\left\langle S_{x}\right\rangle$ for the $X Y$ model in $1 \mathrm{D}$, for $N=100$ spins and with long-range interactions with decay exponent $\alpha=3$. In contrast to the Ising case, DTWA is exact for short times only but can capture longer times than traditional TWA. (b) Time evolution of the spinsqueezing parameter $\xi$. The DTWA gives exact results for short times and good estimates for the achievable $\xi$. (b) Achievable $\xi$ as a function of the filling fraction (averaged over 1000 random configurations). Exact diagonalization for $N<20$, tDMRG otherwise. Inset: Rapid increase of the entanglement entropy for noninteger filling $\bar{n}<1$ (single configuration), rendering tDMRG calculations inefficient in this regime. 
Our results for a 1D chain of spins along the $x$ direction are shown in Fig. 3. In Fig. 3(a), as in the Ising case, first we analyze the evolution of $\left\langle S_{x}\right\rangle$. We compare DTWA and TWA results to an exact tDMRG calculation. Because of the more complicated $X Y$ interactions (the Hamiltonian contains noncommuting terms in contrast to the Ising case), we find that DTWA no longer provides an exact solution. Still, it can capture the evolution on the short time scale $t J \lesssim 0.4$, and significantly improves the traditional TWA result, which, in this example, captures only times $t J \lesssim 0.1$.

In Fig. 3(b), we show the evolution of the spin-squeezing parameter $\xi$ for $\bar{n}=1$. We also find here that DTWA agrees on short times and captures almost all of the spin squeezing that is created in the time evolution. In Fig. 3(b), we plot the maximally achievable spin squeezing as a function of the filling fraction. The DTWA interpolates over the whole range of filling fractions connecting the exactly tractable limits. It is interesting to note that while squeezing implies entanglement (nonseparability) [32], the type of squeezing we consider here is apparently independent of bipartite entanglement, in the sense that for smaller $S_{\mathrm{vN}}\left(\rho_{L}\right)$ we can have large squeezing and vice versa. We note that the connection between entanglement or spin squeezing and discrete phase spaces has been explored in Ref. [40].

\section{Spreading of correlations and transverse field}

In order to understand more systematically what types of correlations can be captured by the DTWA, it is instructive to look at the time evolution of spatial correlations in the system. In Fig. 4, we analyze the time evolution of $\mathcal{C}_{j}^{y y} \equiv\left\langle\hat{\sigma}_{50}^{y} \hat{\sigma}_{50+j}^{y}\right\rangle-\left\langle\hat{\sigma}_{50}^{y}\right\rangle\left\langle\hat{\sigma}_{50+j}^{y}\right\rangle$, i.e., a spatial connected two-point correlation calculated from the center of a system with $N=100$ spins on $M=100$ sites. Again, we consider initially a state with all spins pointing along the $x$ direction. This leads to the fact that initially $\mathcal{C}_{j}^{y y}=0$ for $j \neq 0$. We study how correlations propagate throughout the system $[39,41,42]$ in the presence of long-range interactions (decay exponent $\alpha=3$ ). Besides the Ising and the $X Y$ model, we also treat the case of an Ising interaction with a transverse field (we consider a small $\Omega / J=1$ and a large $\Omega / J=10$ field). The addition of a transverse field to the exactly solvable Ising model adds a noncommuting term in the Hamiltonian and thus enhances the development of quantum correlations during the dynamics. In this situation, one has to resort to tDMRG techniques in order to solve for the full quantum dynamics.

As shown in Fig. 4(a), the dynamics in the pure Ising case is dominated by oscillations. Here, DTWA can capture long-range correlation dynamics $(j \gg 1)$ very well, but the amplitudes and frequencies of, e.g., nearest-neighbor correlations $(j=1)$ are not captured correctly. This can be understood via the error analysis performed in Appendix B [cf. Eq. (B4)]. When adding a transversal field of order $J$ [Fig. 4(b)], the agreement becomes worse. In this case, the total magnetization $S_{z}$ is no longer a conserved quantity and the DTWA fails to capture the propagation of correlations observed in the exact solution. However, as $\Omega$ is increased, the agreement improves and the spreading of correlations starts to show up also in the DTWA solution. Note that in the presence of a large transverse field, the conservation of the total magnetization (now along the transverse field direction) is restored since transitions induced by the Ising
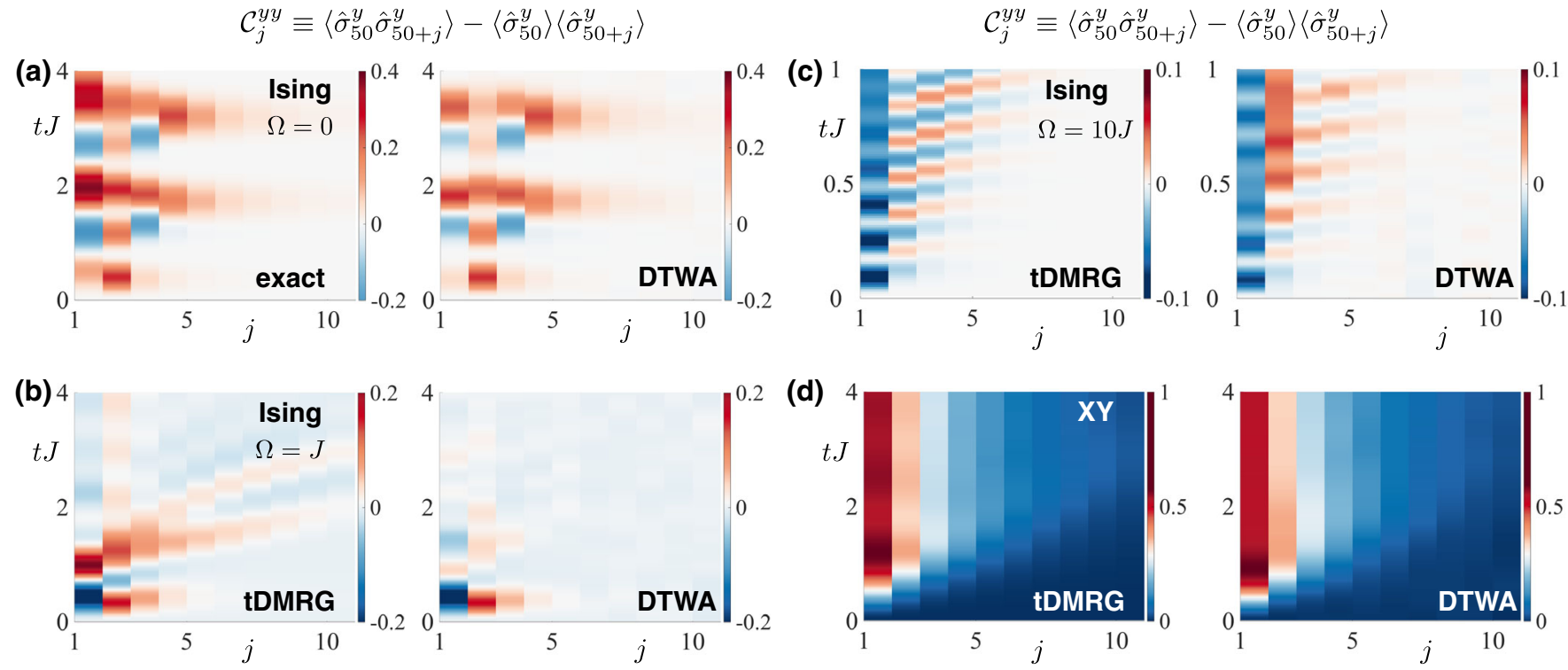

FIG. 4. Spreading of correlations. Time evolution of $\mathcal{C}_{j}^{y y}$ in a system with $N=100$ spins on $M=100$ sites for long-range interactions with decay exponent $\alpha=3$. We consider three models [Eq. (4)]: (a) Ising interactions, (b),(c) Ising interactions plus a transverse field term $\Omega \sum_{i} \hat{\sigma}_{i}^{x}$, and (d) $X Y$ interactions. We compare exact or tDMRG results (left-hand side) with results obtained from the DTWA (righthand side). 

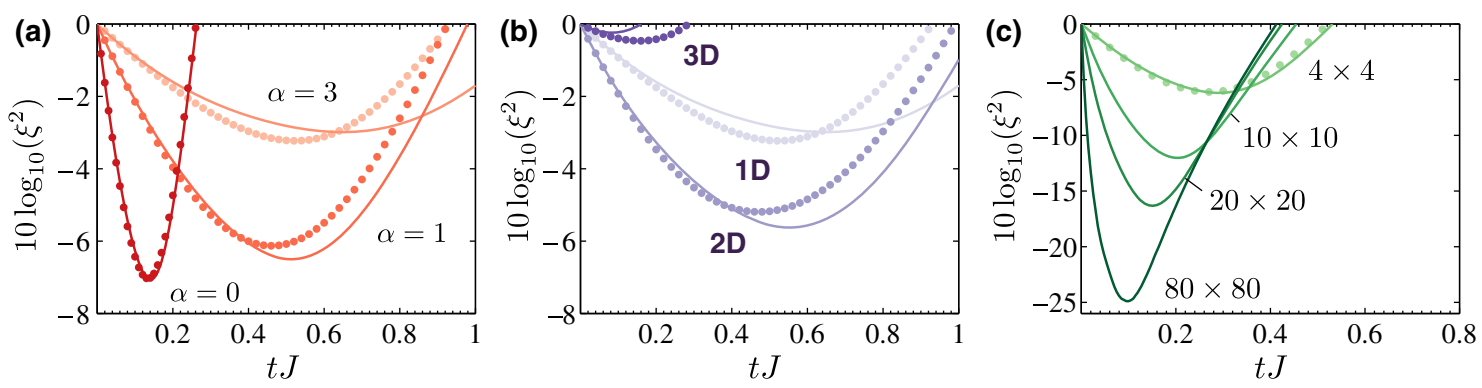

FIG. 5. Range of interactions and higher dimensions ( $X Y$ model). Circles denote exact diagonalization, solid lines DTWA results. The time evolution of the spin-squeezing parameter $\xi$ is shown (a) in $1 \mathrm{D}$ ( $N=20$ spins) for different decay exponents $\alpha$ and (b) with fixed $\alpha=3$ and for different dimensions (1D, $20 \times 1 \times 1$ lattice; $2 \mathrm{D}, 5 \times 4 \times 1 ; 3 \mathrm{D}, 3 \times 3 \times 2)$. With increasing range of interactions, the DTWA improves and spin squeezing increases. (c) DTWA prediction for increasingly large $2 \mathrm{D}$ systems $(N \leq 6400)$ with $\alpha=1$. The exact diagonalization result is shown for the $4 \times 4$ lattice.

term to states that do not preserve it become off resonant. In this large transverse field limit, the dynamics can be mapped back to the dynamics in an effective $X Y$ model [11], and although the DTWA cannot correctly reproduce the oscillation of short-range correlations, it is clearly capable of perfectly reproducing the spreading of $\mathcal{C}_{j}^{y y}$ correlations through the system with time.

The frequencies of the oscillations observed in Figs. 4(a)-4(c) can be understood. Without a transverse field, the exact solution [cf. Eq. (B2)] shows that the correlation functions oscillate with frequencies proportional to the sum of the couplings to the other spins. In the case $\alpha=3$ (short range), the oscillations are dominated by nearest-neighbor couplings. In the case of a strong transverse field $\Omega \gg J$, the spins precess rapidly along the external field and thus the correlation functions oscillate roughly with that rate, $\Omega$. The case $\Omega \sim J$ is most complex since it is close to the critical regime for the ground state. Nevertheless, in this regime the correlations oscillate at a rate $\Omega$, the only energy scale in this case. An interesting question to ask is how dynamics is connected to the ground-state phase transition in this model $[39,43]$. The DTWA could in the future be used for studies in this direction.

The failure of the DTWA to capture the spreading of correlations in generic situations arises from the fact that it formally corresponds to the lowest-order approximation in $\hbar$. Higher-order quantum corrections that induce dynamics in the Wigner distribution can be incorporated via additional "quantum jumps" [18]. Although this is out of the scope of the current work, it is a direction that could lead to further improvement of the DTWA method and should be subjected to further investigation.

\section{Dependence on range of interactions and dimensionality}

We now check the dependence of the validity of the DTWA on the range of interactions and the dimensionality of the system, focusing on the $X Y$ model. The results are summarized in Fig. 5. In Fig. 5(a), we analyze the time evolution of the spin-squeezing parameter $\xi$ for a $N=20$ spin system in 1D ( $M=20$ sites) for various decay exponents $\alpha$ and for fixed $\alpha$ and different dimensions. As expected, we find that longer-ranged interactions lead lo larger amounts of spin squeezing. Remarkably, for longerranged interactions, the increased squeezing is better captured within the DTWA (also when analyzing the relative error). This can be understood in the limit of allto-all interactions $(\alpha=0)$, where, due to the conservation of the total spin, the $X Y$ model becomes equivalent to the Ising model. In that case, we find (Fig. 2) that the DTWA is almost exact. For higher-dimensional systems, we find that the agreement becomes generally better. However, due to the inhomogeneity of the coupling constants, the 3D case is difficult to access since the spin squeezing becomes very small. In Fig. 5(b), we find that for a small 2D system with $\alpha=1$, the agreement is excellent.

\section{CONCLUSION AND OUTLOOK}

The discrete truncated Wigner approximation opens the possibility of computing the dynamics of large spin systems in regimes where currently there is no other theoretical tool at hand [see, in particular, Fig. 5(b)]. This is possible since the computational time for a solution of the mean-field equations only scales polynomially in time. Furthermore, the Monte Carlo sampling can be perfectly parallelized, and the number of required samples for statistical convergence depends only on the observable. Here, this enables us to make quantitative predictions for short-time dynamics in systems with up to 6400 spins [cf. Fig. 5(b)] in two dimensions, a regime that is, for example, clearly inaccessible to tDMRG methods.

The results show that the DTWA is able to capture the buildup of spin squeezing (an entanglement witness [32]). On the other hand, from Eq. (3) and the classical equations of motion (see Appendix A), it follows that $\langle\hat{O}\rangle(t) \approx$ $\sum_{\boldsymbol{\alpha}}^{n_{t}} w_{\alpha}(0) \operatorname{Tr}\left[\hat{O} \otimes_{i=1}^{N} \hat{A}_{\alpha_{i}}^{[i]}(t)\right]$, which resembles the time evolution of an expectation value computed from a separable density matrix. This is not a contradiction, because 
the phase-point operators $\hat{A}_{\alpha}$ are, in fact, not density matrices. While $\operatorname{Tr}\left(\hat{A}_{\alpha}\right)=1$, it is possible to satisfy $\operatorname{Tr}\left(\hat{A}_{\alpha}^{2}\right)>1$; these conditions might be interpreted as a statistical mixture with negative probabilities.

The results we present here demonstrate that the DTWA can be relevant for computing the nonequilibrium dynamics in a variety of recent experimental setups including polar molecules [4,5], Rydberg atoms [6-8], trapped ions [9-12], alkaline earth atoms [44], and solid-state systems, such as nitrogen-vacancy centers [45-47], plasmonic lattices [48], and photonic crystals [49].

It would be interesting to extend the DTWA to deal with open quantum systems and to solve for equilibrium states by employing an evolution in imaginary time. Another direction is to try to combine higher-order corrections to the TWA [18,22] with the idea of discrete phase spaces; this could lead to an even more powerful method capable of capturing quantum many-body dynamics for longer time scales.

\section{ACKNOWLEDGMENTS}

We appreciate useful discussions with K. R. A. Hazzard, A. Polkovnikov, and B. Zhu. This work has been financially supported by JILA-NSF-PFC-1125844, NSF-PIF1211914, ARO, AFOSR, AFOSR-MURI. Computations utilized the Janus supercomputer, supported by NSF (CNS-0821794), NCAR, and CU Boulder/Denver.

\section{APPENDIX A: CLASSICAL EQUATIONS OF MOTION}

To apply the truncated Wigner approximation, we have to compute the classical equations of motion for the spin components of each spin $i: s_{i}^{x}, s_{i}^{y}, s_{i}^{z}$. In the usual phasespace representation of quantum dynamics, for individual spins these can be obtained from the classical Hamiltonian function:

$H_{C}=\frac{1}{2} \sum_{i \neq j}\left[\frac{J_{i j}^{\perp}}{2}\left(s_{i}^{x} s_{j}^{x}+s_{i}^{y} s_{j}^{y}\right)+J_{i j}^{z} s_{i}^{z} s_{j}^{z}\right]+\Omega \sum_{i} s_{j}^{x}$,

via

$$
\dot{s}_{i}^{\alpha}=\left\{s_{i}^{\alpha}, H_{C}\right\}=2 \sum_{\beta} \epsilon_{\alpha \beta \gamma} s_{i}^{\gamma} \frac{\partial H_{C}}{\partial s_{i}^{\beta}},
$$

with $\{.,$.$\} denoting the Poisson bracket and \epsilon$ the fully antisymmetric tensor.

Alternatively, the same equations of motion can be obtained via a product ansatz for the phase-point operators. The exact quantum evolution of any observable $\hat{O}$ is given by $\hat{O}(t)=\hat{U}^{\dagger} \hat{O}(0) \hat{U}$, where $\hat{U}=\exp (-i t \hat{H})$ with $\hat{H}$ the Hamiltonian of the system. The time evolution of a Weyl symbol on the discrete phase space can thus be written as
$\mathcal{O}_{\alpha}^{W}(t)=\operatorname{tr}\left[\hat{O}(t) \hat{\mathcal{A}}_{\boldsymbol{\alpha}}\right] / 2=\operatorname{tr}\left[\hat{O}(0) \hat{U} \hat{\mathcal{A}}_{\boldsymbol{\alpha}} \hat{U}^{\dagger}\right] / 2$, where we use the cyclic invariance under the trace. Thus, in order to calculate $\mathcal{O}_{\alpha}^{W}(t)$, we can evolve the many-body phasepoint operator according to the von Neumann equation $d \hat{\mathcal{A}}_{\boldsymbol{\alpha}} / d t=-i\left[\hat{H}, \hat{\mathcal{A}}_{\boldsymbol{\alpha}}\right]$. Making a product ansatz for the phase-point operators, $\hat{\mathcal{A}}_{\boldsymbol{\alpha}} \approx \hat{A}_{\alpha_{1}}^{[1]} \otimes \hat{A}_{\alpha_{2}}^{[2]} \otimes \ldots \otimes \hat{A}_{\alpha_{M}}^{[M]}$, and assuming a general parametrization, $\hat{A}_{\alpha_{i}}^{[i]}\left[\mathbf{r}_{i}(t)\right]=\wp_{\wp}\left[\mathbf{r}_{i}(t)\right]$, yields a coupled set of differential equations for $\mathbf{r}_{i}(t) \equiv$ $\left(s_{i}^{x}, s_{i}^{y}, s_{i}^{z}\right)^{T}$.

For example, for the Ising interaction Hamiltonian,

$$
H_{Z Z}=\frac{1}{2} \sum_{n, m} J_{n m} \sigma_{n}^{z} \sigma_{m}^{z}
$$

with $J_{n m}=J_{m n}$ and $J_{n n}=0$, the classical (mean-field) equations for the spin components are then given by

$$
\begin{gathered}
\dot{s}_{n}^{x}=-2 s_{n}^{y} \sum_{m} J_{n, m}^{z} s_{m}^{z} \equiv-2 s_{n}^{y} \beta_{n}^{z}, \\
\dot{s}_{n}^{y}=2 s_{n}^{x} \sum_{m} J_{n, m}^{z} s_{m}^{z} \equiv 2 s_{n}^{x} \beta_{n}^{z}, \\
\dot{s}_{n}^{z}=0,
\end{gathered}
$$

where we introduce the quantity $\beta_{n}^{\alpha=x, y, z} \equiv \sum_{m} J_{n, m}^{z} s_{m}^{\alpha=x, y, z}$, which can be interpreted as an effective magnetic field on spin $n$ induced by the mean-field interactions with the other spins. Solving these equations yields

$$
s_{n}^{ \pm}(t)=s_{n}^{ \pm}(0) \exp \left( \pm 2 i t \sum_{j} J_{n j} s_{j}^{z}\right),
$$

where $s^{ \pm}=\left(s_{n}^{x} \pm i s_{n}^{y}\right) / 2$. For completeness, we also give the classical equations of motion that are used for the $X Y$ interaction in Eq. (4),

$$
\dot{s}_{n}^{x}=s_{n}^{z} \sum_{m} J_{n, m}^{\perp} s_{m}^{y} \equiv s_{n}^{z} \beta_{n}^{y},
$$

$$
\begin{gathered}
\dot{s}_{n}^{y}=-s_{n}^{z} \sum_{m} J_{n, m}^{\perp} s_{m}^{x} \equiv-s_{n}^{z} \beta_{n}^{x}, \\
\dot{s}_{n}^{z}=\sum_{m} J_{n, m}^{\perp}\left(s_{m}^{x} s_{n}^{y}-s_{m}^{y} s_{n}^{x}\right) \equiv s_{n}^{y} \beta_{n}^{x}-s_{n}^{x} \beta_{n}^{y},
\end{gathered}
$$

and for dynamics under a transverse field,

$$
\begin{gathered}
\dot{s}_{n}^{y}=-2 \Omega s_{n}^{z}, \\
\dot{s}_{n}^{z}=2 \Omega s_{n}^{y} .
\end{gathered}
$$




\section{APPENDIX B: CORRELATION FUNCTIONS IN THE ISING MODEL}

The time evolution for the Ising Hamiltonian [Eq. (A3)] can be solved exactly [28-30]. For a local operator at site $k\left[\sigma_{k}^{ \pm}=\left(\sigma_{k}^{x} \pm i \sigma_{k}^{y}\right) / 2\right]$, the time evolution can be calculated as

$$
\left\langle\sigma_{k}^{ \pm}\right\rangle(t)=\frac{\left\langle\sigma_{k}^{ \pm}\right\rangle(0)}{2^{N}} \sum_{\substack{m_{1}, m_{N} \\ \in\{-1,+1\}}} \exp \left( \pm 2 i t \sum_{j=1}^{N} J_{k j} m_{j}\right) .
$$

Here, each $m_{i}$ takes the values -1 and +1 , and the sum runs over all $2^{N}$ possible combinations. Comparing with Eq. (A7), one sees that DTWA gives the exact time evolution in this case, when the sum is approximated via a random sampling of $s^{z}$ taking the values $+1,-1$ (see discussion in the main text).

The same calculation is possible for correlations. For example, between particle $i$ and $j(i<j)$,

$$
\begin{aligned}
\left\langle\sigma_{i}^{ \pm} \sigma_{j}^{ \pm}\right\rangle(t)_{\mathrm{exact}}= & \frac{\left\langle\sigma_{i}^{ \pm} \sigma_{j}^{ \pm}\right\rangle(0)}{2^{N-2}} \sum_{m_{1} \ldots m_{i-1} m_{i+1} \cdot m_{j-1} m_{j+1} \ldots m_{N}} \in\{-1,+1\} \\
& \times \exp \left( \pm 2 i t \sum_{\substack{a=1 \\
a \neq i, j}}^{N} J_{i a} m_{a}\right) \\
& \times \exp \left( \pm 2 i t \sum_{\substack{b=1 \\
b \neq i, j}}^{N} J_{j b} m_{b}\right)
\end{aligned}
$$

Note that the two sums with $m_{i}$ and $m_{j}$ are missing. This is to be compared with the DTWA, which estimates the same quantity (in the limit $n_{t} \rightarrow \infty$ ) as

$$
\begin{aligned}
\left\langle\sigma_{i}^{ \pm} \sigma_{j}^{ \pm}\right\rangle(t)_{\mathrm{DTWA}}= & \frac{\left\langle\sigma_{i}^{ \pm} \sigma_{j}^{ \pm}\right\rangle(0)}{2^{N}} \sum_{s_{1}^{z} \ldots s_{N}^{z} \in\{-1,+1\}} \\
& \times \exp \left( \pm 2 i t \sum_{a=1}^{N} J_{i a} s_{a}^{z}\right) \\
& \times \exp \left( \pm 2 i t \sum_{b=1}^{N} J_{j b} s_{b}^{z}\right)
\end{aligned}
$$

We note that Eqs. (B2) and (B3) are valid for all combinations of signs,,+++--+ , and -- . One sees that the only difference between Eqs. (B2) and (B3) is the two additional sums with $s_{i}^{z}, s_{j}^{z} \in\{-1,+1\}$ in Eq. (B3). This gives rise to an error of

$$
\left\langle\sigma_{i}^{ \pm} \sigma_{j}^{ \pm}\right\rangle(t)_{\mathrm{DTWA}}=\left\langle\sigma_{i}^{ \pm} \sigma_{j}^{ \pm}\right\rangle(t)_{\mathrm{exact}} \cos ^{2}\left(2 t J_{i j}\right) .
$$

Since, for example, $\left\langle S_{x}^{2}\right\rangle / N^{2}=\frac{1}{N}+\frac{1}{N^{2}} \sum_{i \neq j}\left(\left\langle\sigma_{i}^{+} \sigma_{j}^{+}\right\rangle+\right.$ $\left\langle\sigma_{i}^{+} \sigma_{j}^{-}\right\rangle+$c.c. $)$, this correlation contains an error. Analogously, it is straightforward to see that, due to the conservation of the $z$ component of the spin, there is no error made when calculating $\operatorname{Re}\left\langle S_{z} S_{y}\right\rangle=\sum_{i \neq j} 2 \operatorname{Re}\left\langle\sigma_{j}^{z} \sigma_{i}^{-}\right\rangle$ with DTWA.

\section{APPENDIX C: STATISTICAL CONVERGENCE}

The quantum noise in the DTWA calculations is introduced via a Monte Carlo sampling. For the success of the method in large systems, it is important that the results converge when increasing the number of sample trajectories $n_{t}$. We test, for example, the time evolution of $S_{x}$ and the squeezing parameter for different numbers of trajectories $n_{t}$, for a small system with $X Y$ interactions $(\alpha=1)$ and for a large system (see Fig. 6). In all cases, for $n_{t}>4000$ the curves are essentially indistinguishable. The maximum relative difference from the $n_{t}=64000$ result in these calculations decreases as $1 / \sqrt{n_{t}}$, as expected, and has the same magnitude for both the small and the large system. For the one-particle observable $S_{x}$, the convergence with increasing $n_{t}$ is faster than for the squeezing parameter, a two-particle observable.
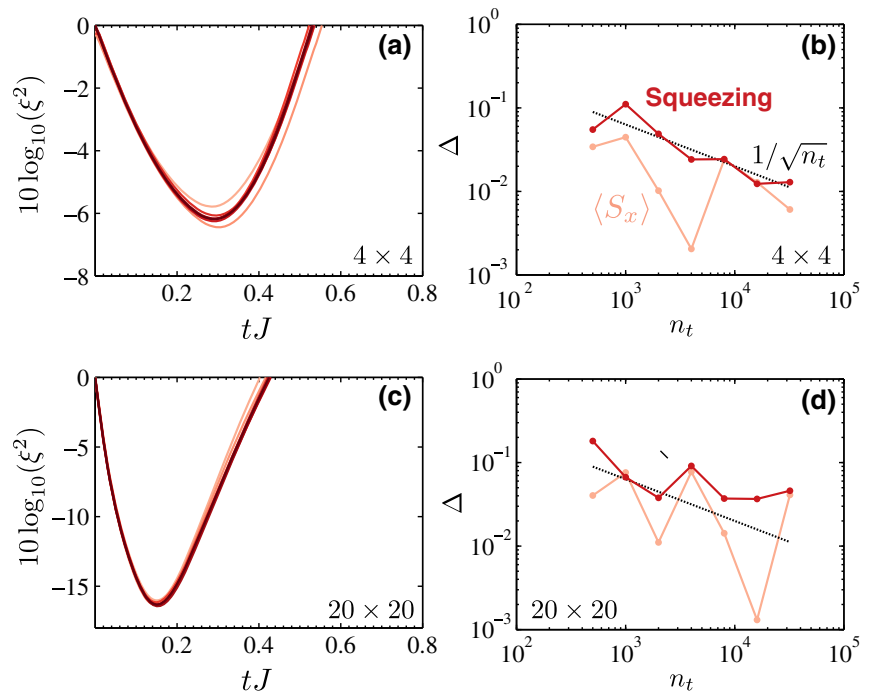

FIG. 6. Statistical convergence. (a),(c) Time evolution of the squeezing parameter in DTWA for different number of trajectories: $n_{t}=500,1000,2000,4000,8000,16000$, 32000,64000 from light to dark. (a) Small $4 \times 4$ system with $X Y$ interactions $(\alpha=1)$. (c) $20 \times 20$ system. In both cases, for $n_{t}>4000$, curves are essentially indistinguishable. Panels (b),(d) show the maximum relative difference of the calculations from the $n_{t}=64000$ result. The upper line is for the spinsqueezing parameter, the lower line for $\left\langle S_{x}\right\rangle$; there are smaller relative differences for this simple observable. Both differences decrease as $1 / \sqrt{n_{t}}$ (dashed line) as expected. Panels (b) and (d) are for the small and large system, respectively. 
[1] I. Bloch, J. Dalibard, and S. Nascimbène, Quantum Simulations with Ultracold Quantum Gases, Nat. Phys. 8, 267 (2012).

[2] R. Blatt and C. F. Roos, Quantum Simulations with Trapped Ions, Nat. Phys. 8, 277 (2012).

[3] A. Aspuru-Guzik and P. Walther, Photonic Quantum Simulators, Nat. Phys. 8, 285 (2012).

[4] B. Yan, S. A. Moses, B. Gadway, J. P. Covey, K. R. A. Hazzard, A. M. Rey, D. S. Jin, and J. Ye, Observation of Dipolar Spin-Exchange Interactions with Lattice-Confined Polar Molecules, Nature (London) 501, 521 (2013).

[5] K. R. A. Hazzard, B. Gadway, M. Foss-Feig, B. Yan, S. A. Moses, J. P. Covey, N. Y. Yao, M. D. Lukin, J. Ye, D. S. Jin, and A. M. Rey, Many-Body Dynamics of Dipolar Molecules in an Optical Lattice, Phys. Rev. Lett. 113, 195302 (2014).

[6] R. Löw, H. Weimer, J. Nipper, J. B. Balewski, B. Butscher, H. P. Büchler, and T. Pfau, An Experimental and Theoretical Guide to Strongly Interacting Rydberg Gases, J. Phys. B 45, 113001 (2012).

[7] P. Schauß, M. Cheneau, M. Endres, T. Fukuhara, S. Hild, A. Omran, T. Pohl, C. Gross, S. Kuhr, and I. Bloch, Observation of Spatially Ordered Structures in a TwoDimensional Rydberg Gas, Nature (London) 491, 87 (2012).

[8] P. Schauß, J. Zeiher, T. Fukuhara, S. Hild, M. Cheneau, T. Macrì, T. Pohl, I. Bloch, and C. Gross, Dynamical Crystallization in a Low-Dimensional Rydberg Gas, arXiv: 1404.0980.

[9] R. Islam, C. Senko, W. C. Campbell, S. Korenblit, J. Smith, A. Lee, E. E. Edwards, C.-C. J. Wang, J. K. Freericks, and C. Monroe, Emergence and Frustration of Magnetism with Variable-Range Interactions in a Quantum Simulator, Science 340, 583 (2013).

[10] P. Jurcevic, B. P. Lanyon, P. Hauke, C. Hempel, P. Zoller, R. Blatt, and C. F. Roos, Observation of Entanglement Propagation in a Quantum Many-Body System, Nature (London) 511, 202 (2014).

[11] P. Richerme, Z.-X. Gong, A. Lee, C. Senko, J. Smith, M. Foss-Feig, S. Michalakis, A. V. Gorshkov, and C. Monroe, Non-Local Propagation of Correlations in Long-Range Interacting Quantum Systems, Nature (London) 511, 198 (2014).

[12] J. W. Britton, B. C. Sawyer, A. C. Keith, C. C. Wang, J. K. Freericks, H. Uys, M. J. Biercuk, and J. J. Bollinger, Engineered Two-Dimensional Ising Interactions in a Trapped-Ion Quantum Simulator with Hundreds of Spins, Nature (London) 484, 489 (2012).

[13] G. Vidal, Efficient Simulation of One-Dimensional Quantum Many-Body Systems, Phys. Rev. Lett. 93, 040502 (2004).

[14] S. R. White and A. E. Feiguin, Real-Time Evolution Using the Density Matrix Renormalization Group, Phys. Rev. Lett. 93, 076401 (2004).

[15] A. J. Daley, C. Kollath, U. Schollwöck, and G. Vidal, TimeDependent Density-Matrix Renormalization-Group Using Adaptive Effective Hilbert Spaces, J. Stat. Mech. Theory Exp. (2004) P04005.

[16] A. Kamenev, Many-Body Theory of Non-Equilibrium Systems, arXiv:cond-mat/0412296.

[17] P. B. Blakie, A. S. Bradley, M. J. Davis, R. J. Ballagh, and C. W. Gardiner, Dynamics and Statistical Mechanics of
Ultra-Cold Bose Gases Using c-Field Techniques, Adv. Phys. 57, 363 (2008).

[18] A. Polkovnikov, Phase Space Representation of Quantum Dynamics, Ann. Phys. (Amsterdam) 325, 1790 (2010).

[19] A Altland, V. Gurarie, T. Kriecherbauer, and A. Polkovnikov, Nonadiabaticity and Large Fluctuations in a Many-Particle Landau-Zener Problem, Phys. Rev. A 79, 042703 (2009).

[20] W. K. Wootters, A Wigner-Function Formulation of FiniteState Quantum Mechanics, Ann. Phys. (N.Y.) 176, 1 (1987).

[21] U. Fano, Description of States in Quantum Mechanics by Density Matrix and Operator Techniques, Rev. Mod. Phys. 29, 74 (1957).

[22] S. M. Davidson and A. Polkovnikov, SU(3) Semiclassical Representation of Quantum Dynamics of Interacting Spins, Phys. Rev. Lett. 114, 045701 (2015).

[23] L. Cohen and M. O. Scully, Joint Wigner Distribution for Spin-1/2 Particles, Found. Phys. 16, 295 (1986).

[24] Y. Takahashi and F. Shibata, Spin Coherent State Representation in Non-Equilibrium Statistical Mechanics, J. Phys. Soc. Jpn. 38, 656 (1975).

[25] W. K. Wootters, Picturing Qubits in Phase Space, arXiv: quant-ph/0306135.

[26] A. de Paz, A. Sharma, A. Chotia, E. Maréchal, J. H. Huckans, P. Pedri, L. Santos, O. Gorceix, L. Vernac, and B. Laburthe-Tolra, Nonequilibrium Quantum Magnetism in a Dipolar Lattice Gas, Phys. Rev. Lett. 111, 185305 (2013).

[27] J. Nipper, J. B. Balewski, A. T. Krupp, S. Hofferberth, R. Löw, and T. Pfau, Atomic Pair-State Interferometer: Controlling and Measuring an Interaction-Induced Phase Shift in Rydberg-Atom Pairs, Phys. Rev. X 2, 031011 (2012).

[28] I. J. Lowe and R. E. Norberg, Free-Induction Decays in Solids, Phys. Rev. 107, 46 (1957).

[29] M. Foss-Feig, K. R. A. Hazzard, J. J. Bollinger, and A. M. Rey, Nonequilibrium Dynamics of Arbitrary-Range Ising Models with Decoherence: An Exact Analytic Solution, Phys. Rev. A 87, 042101 (2013).

[30] M. van den Worm, B. C. Sawyer, J. J. Bollinger, and M. Kastner, Relaxation Timescales and Decay of Correlations in a Long-Range Interacting Quantum Simulator, New J. Phys. 15, 083007 (2013).

[31] M. R. Hush, A. R. R. Carvalho, and J. J. Hope, NumberPhase Wigner Representation for Efficient Stochastic Simulations, Phys. Rev. A 81, 033852 (2010).

[32] A. Sørensen, L.-M. Duan, J. I. Cirac, and P. Zoller, ManyParticle Entanglement with Bose-Einstein Condensates, Nature (London) 409, 63 (2001).

[33] V. Giovannetti, S. Lloyd, and L. Maccone, Advances in Quantum Metrology, Nat. Photonics 5, 222 (2011).

[34] D. J. Wineland, J. J. Bollinger, W. M. Itano, F. L. Moore, and D. J. Heinzen, Spin Squeezing and Reduced Quantum Noise in Spectroscopy, Phys. Rev. A 46, R6797 (1992).

[35] M. Kitagawa and M. Ueda, Squeezed Spin States, Phys. Rev. A 47, 5138 (1993).

[36] L. Amico, R. Fazio, A. Osterloh, and V. Vedral, Entanglement in Many-Body Systems, Rev. Mod. Phys. 80, 517 (2008).

[37] J. Eisert, M. Cramer, and M. B. Plenio, Colloquium: Area Laws for the Entanglement Entropy, Rev. Mod. Phys. 82, 277 (2010). 
[38] A. J. Daley, H. Pichler, J. Schachenmayer, and P. Zoller, Measuring Entanglement Growth in Quench Dynamics of Bosons in an Optical Lattice, Phys. Rev. Lett. 109, 020505 (2012).

[39] J. Schachenmayer, B. P. Lanyon, C. F. Roos, and A. J. Daley, Entanglement Growth in Quench Dynamics with Variable Range Interactions, Phys. Rev. X 3, 031015 (2013).

[40] M. A. Marchiolli, D. Galetti, and T. Debarba, Spin Squeezing and Entanglement via Finite-Dimensional Discrete Phase-Space Description, Int. J. Quantum. Inform. 11, 1330001 (2013).

[41] J. Eisert, M. van den Worm, S. R. Manmana, and M. Kastner, Breakdown of Quasilocality in Long-Range Quantum Lattice Models, Phys. Rev. Lett. 111, 260401 (2013).

[42] Z.-X. Gong, M. Foss-Feig, S. Michalakis, and A. V. Gorshkov, Persistence of Locality in Systems with PowerLaw Interactions, Phys. Rev. Lett. 113, 030602 (2014).

[43] A. M. Rey, V. Gritsev, I. Bloch, E. Demler, and M. D. Lukin, Preparation and Detection of Magnetic Quantum Phases in Optical Superlattices, Phys. Rev. Lett. 99, 140601 (2007).
[44] M. J. Martin, M. Bishof, M. D. Swallows, X. Zhang, C. Benko, J. von Stecher, A. V. Gorshkov, A. M. Rey, and Jun Ye, A Quantum Many-Body Spin System in an Optical Lattice Clock, Science 341, 632 (2013).

[45] M. W. Doherty, N. B. Manson, P. Delaney, F. Jelezko, J. Wrachtrup, and L. C. L. Hollenberg, The Nitrogen-Vacancy Colour Centre in Diamond, Phys. Rep. 528, 1 (2013).

[46] F. Dolde, I. Jakobi, B. Naydenov, N. Zhao, S. Pezzagna, C. Trautmann, J. Meijer, P. Neumann, F. Jelezko, and J. Wrachtrup, Room-Temperature Entanglement between Single Defect Spins in Diamond, Nat. Phys. 9, 139 (2013).

[47] J. R. Weber, W. F. Koehl, J. B. Varley, A. Janotti, B. B. Buckley, C. G. Van de Walle, and D. D. Awschalom, Quantum Computing with Defects, Proc. Natl. Acad. Sci. U.S.A. 107, 8513 (2010).

[48] M. Gullans, T. G. Tiecke, D. E. Chang, J. Feist, J. D. Thompson, J. I. Cirac, P. Zoller, and M. D. Lukin, Nanoplasmonic Lattices for Ultracold Atoms, Phys. Rev. Lett. 109, 235309 (2012).

[49] A. González-Tudela, C.-L. Hung, D. E. Chang, J. I. Cirac, and H. J. Kimble, Subwavelength Vacuum Lattices and Atom-Atom Interactions in Photonic Crystals, arXiv: 1407.7336. 